\title{
Hacia una sociología del libro y la lectura en el Perú: consumo cultural y distinción social en el mercado de libros Amazonas ${ }^{1}$
}

\author{
Rodrigo Canelo
}

doi: $10.46476 /$ ra.v2i2.94

\section{Resumen}

El mercado de libros en el jirón Amazonas (Lima), conocido también como la «Feria de libros Amazonas», no es solo un lugar en donde se compran libros según una racionalidad económica, sino que también es un espacio que presenta una experiencia de consumo cultural donde se expresan los valores simbólicos que se suelen atribuir al libro y la lectura. La pregunta que guio esta investigación fue la siguiente: ¿Por qué los libros y la lectura son importantes y necesarios para los agentes en el mercado de libros del jirón Amazonas?

Este es un estudio de caso que planteó un diseño de investigación mixto, el cual se sostiene en 29 entrevistas, una encuesta representativa a los visitantes del mercado de libros y una experiencia etnográfica de observación y participación. La investigación encontró que los discursos sobre el libro y la lectura tienen un efecto importante en las relaciones sociales de los agentes, pero también en el sentido con el que practican la lectura o dejan de hacerlo

Palabras clave: lectura, cultura, consumo cultural, educación, distinción social.

1. Tesis para optar el título de Licenciado en Sociología por la Pontificia Universidad Católica del Perú. Disponible en: https://tesis.pucp.edu.pe/repositorio/handle/20.500.12404/18542 
En el Perú, hay dos grandes mitos instalados en la opinión pública respecto al libro y la lectura que se reproducen de manera cotidiana y se sostienen en la falta de información y en las dificultades educativas y económicas que impiden acceder, entender y disfrutar de los libros. De manera general, muchas personas consideran (i) que las y los peruanos no leen y (ii) que la lectura de libros es una práctica relegada a las clases altas.

Hace más de 20 años, Carmen Villanueva, quien fue directora del sistema de bibliotecas de la Pontificia Universidad Católica del Perú, expresó su preocupación frente a un problema que podría considerarse como vigente hasta la fecha: «Es un lugar común decir que en el Perú no se lee. Si queremos ser realmente exactos, deberíamos decir que no sabemos qué se lee, ni cuánto, ni quiénes ni por qué se lee o no se lee» (Villanueva, 1997).

Desde la sociología, esta investigación buscó contribuir con algunas ideas al problema comentado por Villanueva y propuso un acercamiento al libro y la lectura desde el sentido y el valor que adquieren en un universo de significación específico: la Feria de libros del jirón Amazonas en el centro de Lima, un mercado «popular» en donde circulan bienes de la «alta cultura». Enfocarse en el sentido y el valor que adquieren los libros y la lectura permitió a esta investigación entender las razones objetivas (como adquirir mayores conocimientos, habilidades o experiencias estéticas) y subjetivas (como el prestigio, la legitimidad o la «liberación personal») que motivan la lectura.

La «Feria de libros del jirón Amazonas» (de aquí en adelante, Amazonas) expresa uno de los muchos rostros de la desigualdad en el país y reta directamente los dos mitos comentados anteriormente. Este mercado de libros tiene más de 20 años de funcionamiento en su local actual, cuenta con 200 puestos de venta y ofrece a sus visitantes una biblioteca gratuita, además de diversas actividades culturales. Por un lado, el mercado Amazonas permite que miles de peruanos y peruanas accedan a libros económicos, por otro lado, es un espacio en donde ocurren intercambios simbólicos respecto a lo que se piensa de los libros, la lectura, la educación y la cultura. Por estas razones, este lugar es ideal para iniciar una aún ausente reflexión sociológica del libro y la lectura en el Perú. 


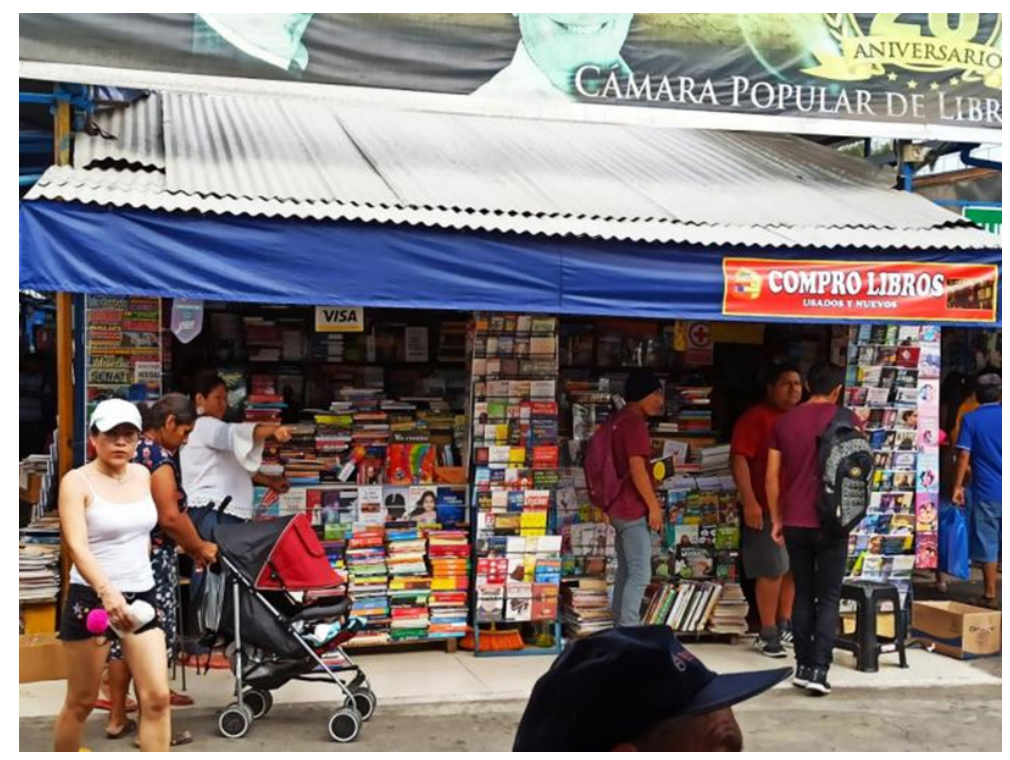

Imagen 1. Fuente: Elaboración propia

La presente investigación problematizó esta forma de consumo cultural y adoptó una mirada crítica a aquello que se hace, dice y piensa respecto a libros y lectura. Aquí se prestó atención a como las estructuras sociales externas (sistema educativo) tienen una relación estrecha con las estructuras mentales internas (ideas, deseos y prácticas). De esta manera, la pregunta de investigación fue la siguiente: ¿Por qué los libros y la lectura son importantes y necesarios para los agentes en el mercado de libros del jirón Amazonas?

Esta pregunta de investigación fue respondida de la siguiente manera: El mercado de libros en el jirón Amazonas es un lugar en donde se expresan las disposiciones e ideas propias de una «sociedad escolarizada» (David Baker, 2014), que posiciona a los libros y la lectura como valores simbólicos fundamentales para insertarse satisfactoriamente en la sociedad. Los libros y la lectura son importantes en Amazonas, no por el lugar en sí mismo, sino por un modelo de sociedad en donde las aspiraciones, los proyectos de vida y los intereses de los agentes se encuentran orientados hacia la educación. Estas disposiciones e ideas presentes en la estructura social son reproducidas de manera individual por los agentes casi de forma natural y sin ser percibidas como arbitrarias. Lectores y no lectores en un lugar como Amazonas, en tanto han tenido una experiencia de formación educativa formal, comparten, aunque con diferencias, las creencias y proyectos de la sociedad escolarizada.

La base teórica de esta investigación está en la sociología de la cultura y de la educación. En tanto, esta investigación se centró en los procesos sociales de significación, los trabajos de autores como García Canclini, Bourdieu, Lahire y Baker guiaron este análisis. A partir del concepto de la «sociedad escolarizada» de Baker, esta investigación elaboró el concepto de «habitus escolar» sobre la base 
del habitus bourdiano, el cual alude a una disposición inconsciente y un interés objetivo por los libros y la lectura, los cuales son los capitales culturales que, en la sociedad escolarizada, conceden prestigio y distinción social.

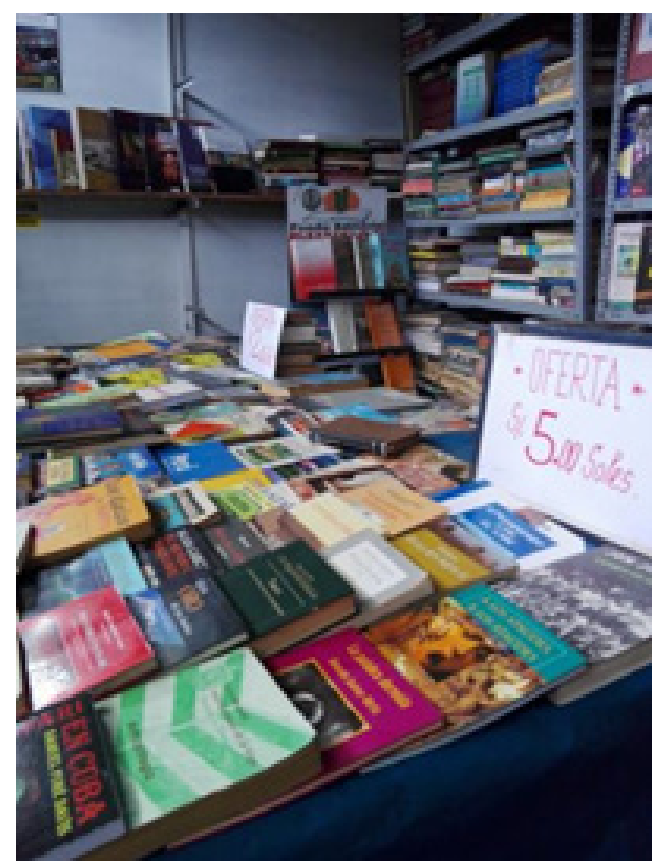

Imagen 2

Fuente: Elaboración propia

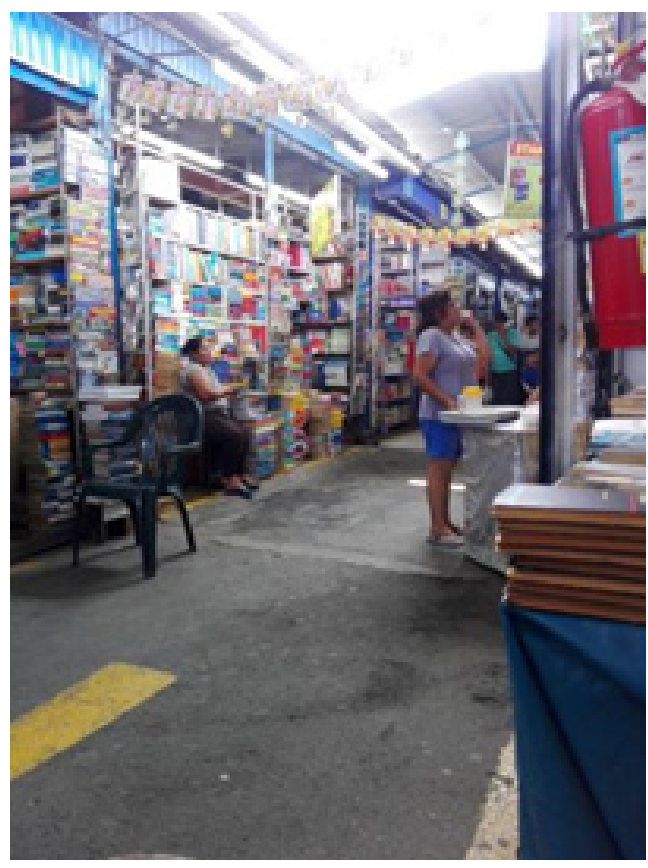

Imagen 3

Fuente: Elaboración propia

Este es un estudio de caso que planteó un diseño de investigación mixto y empleó métodos cualitativos y cuantitativos que permitieron acercarse a quienes visitan el mercado y a quienes venden libros en él. Esta investigación se sostiene en 29 entrevistas semiestructuradas (11 exploratorias y 18 a profundidad), una encuesta representativa a los y las visitantes del mercado (399 personas) y un periodo de observación y participación etnográfica en la que el investigador ayudó como vendedor de libros en un puesto del mercado. Esta forma de aproximarse a la investigación permitió un diálogo entre cuerpos de información distintos, lo cual propició una lectura «cualitativa» de los datos «cuantitativos» y viceversa.

Los hallazgos de la investigación se presentaron en tres capítulos. El primer capítulo se tituló «Amazonas como mercado de libros: historia y características» donde se explicó por qué este mercado es valioso. Para esto, se identificó a los agentes en este espacio y se expuso su relación con este lugar. La encuesta de la investigación señaló que:

- $50.9 \%$ de los visitantes tienen 30 años o menos.

- $56.5 \%$ de los visitantes son hombres y $43.5 \%$ mujeres.

- 77\% ha cursado algún tipo de educación superior. 
- 4 de cada 10 visitantes son estudiantes o educadores.

- $75.1 \%$ percibe ingresos familiares mensuales menores a 3001 soles y $10.8 \%$ menos de 1001 soles.

Los visitantes se dividen en dos grupos: ocasionales y habituales. Cada grupo tiene subgrupos, los cuales visitan el mercado y se aproximan a la lectura de manera diferente (por cumplir obligaciones académicas, conseguir lecturas para sus hijos, conversar con los libreros, disfrutar de la lectura o simplemente visitar este lugar). Por el lado de quienes expenden libros, se encuentran los vendedores de libros y los libreros. Los vendedores suelen ser personas jóvenes que no practican la lectura y solo conocen los libros «de la tapa hacia afuera»; ellos son básicamente comerciantes. Los libreros suelen ser hombres mayores de 50 años que disfrutan de la lectura, conocen los libros «de la tapa hacia adentro» y consideran que su labor aporta a la sociedad; además, estos últimos suelen estar en contra de la piratería.

Si bien existen diferentes tipos de agentes, hay cuatro grandes ideas sobre el mercado que están generalizadas. De esta forma, Amazonas es un lugar en donde:

1. Se consiguen libros a buen precio.

2. Hay una gran variedad de libros.

3. Uno puede entretenerse y pasar un buen rato.

4. Hay «cultura».

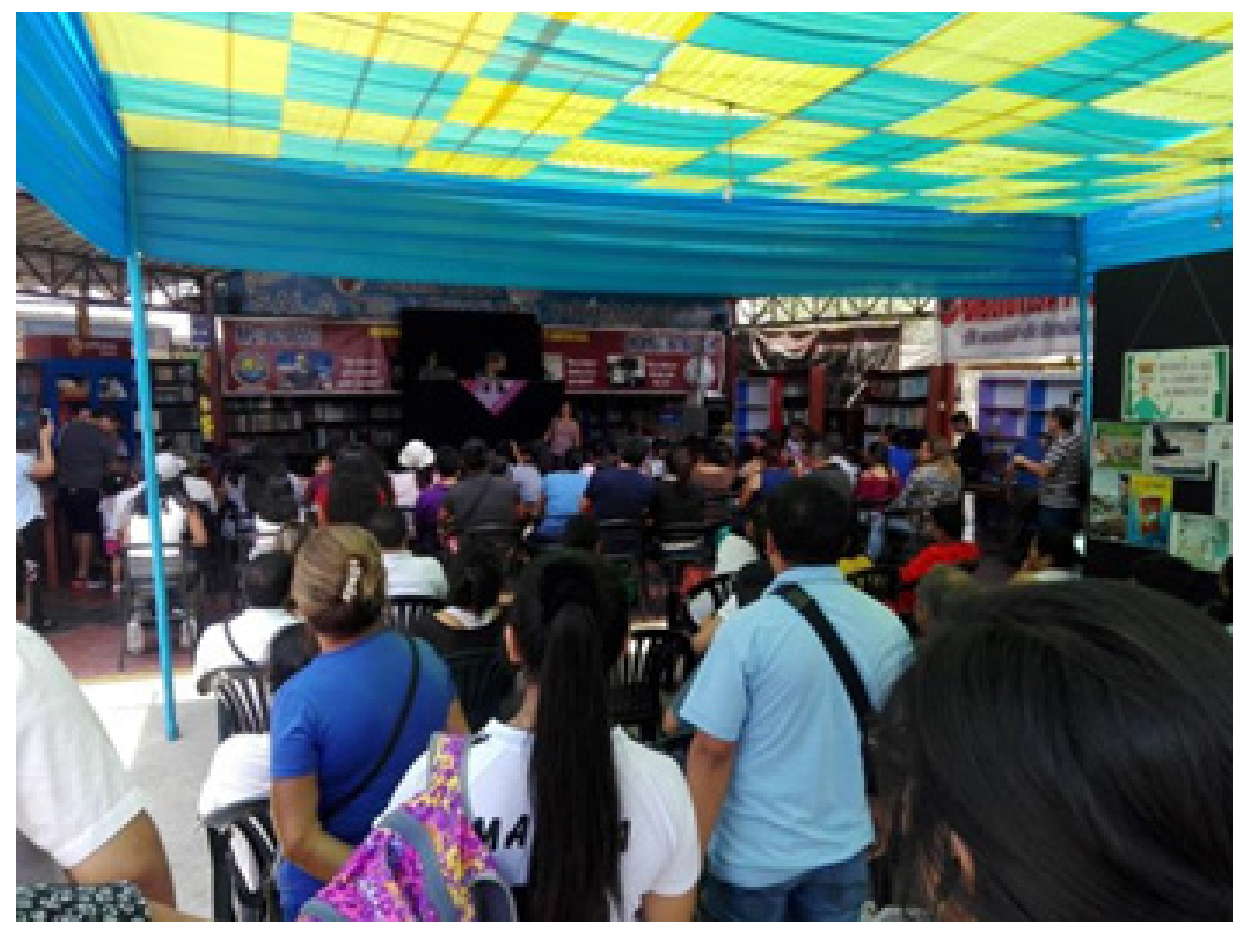

Imagen 4. Fuente: Elaboración propia 
Esta investigación sostuvo que Amazonas es importante para los agentes porque, por un lado, brinda una experiencia de consumo cultural que legitima los intereses e ideas de los agentes respecto a los libros, la lectura y la educación; por otro lado, permite a personas con escasos recursos económicos, ejecutar estrategias personales para afrontar los retos de la sociedad escolarizada y obtener las oportunidades que esta les presenta.

El segundo capítulo se tituló «La relación de los agentes con los libros y la lectura» y explicó cómo se articula lo «social» de la estructura y lo «individual» de los agentes en la práctica de la lectura. En este capítulo se expuso cómo la lectura no es y no depende solamente de una capacidad individual, sino que se ve afectada por la ubicación del agente en un contexto material y social particular, que facilita o no el acceso al libro, al tiempo para leer y que reúne a agentes e instituciones que generan una motivación por leer o fuerzan a hacerlo.

Para poder leer, una persona necesita (i) aprender esta habilidad y (ii) tener algún interés o necesidad de utilizarla, es decir, un sentido que justifique practicarla. En tanto la lectura es un capital cultural incorporado, debe ser inculcado y requiere de tiempo y esfuerzo individual, pero también del apoyo de una persona que domine esta habilidad. La escuela suele ser la institución que enseña esta habilidad, sin embargo, suele generar lectores por obligación. El interés «legítimo» por leer es desarrollado, por lo general, en los espacios en donde los agentes comparten con familiares, amigos o profesores específicos que disfrutan de la lectura y que lograron compartir este interés.

Este capítulo encontró que aproximadamente 8 de cada 10 visitantes señalan que leen al menos una vez por semana y que el promedio de libros leídos por año es de 8.22 libros. Estos datos son mucho mayores a los del resto del Perú según el IOP (2015), el cual señala que 4 de cada 10 peruanos lee libros al menos 1 vez por semana y que el promedio nacional de libros leídos por año es 3.3. Si bien esta información es útil, debe ser tomada con precaución, puesto que podría pasar por alto la heterogeneidad en las formas en que se practica la lectura.

Por un lado, estos datos podrían no considerar cómo el deseo de no ser percibido negativamente altera las respuestas sobre los hábitos de lectura personales, por otro lado, preguntar solo por la frecuencia de lectura o el número de libros leídos por año, podría llevar a pensar que, por ejemplo, las personas que no completan ni un libro al año (como los estudiantes que solo leen libros por capítulos) son personas que no leen en lo absoluto. Además, estas preguntas no pueden asegurar que todas las personas comparten la misma noción de «libro» ni pueden evaluar la calidad, el tiempo o el contexto de lectura. Por estas dificultades, esta investigación se centró solo en las intenciones por las que se lee e identificó motivos individuales explícitos y motivos sociales implícitos. 
El tercer capítulo se tituló «El valor del libro y la lectura» y explicó cómo se identifica y cuál es el valor simbólico de los libros y la lectura en el mercado Amazonas. Este capítulo expuso los discursos e ideas que subyacen a los motivos por los que se lee y explicó que el mercado Amazonas es una de las «arenas de juego» de la sociedad escolarizada. En ese sentido, aquello que ahí orienta a los agentes al libro y la lectura, probablemente es lo mismo que orienta a otros agentes en otros espacios a estos tipos de capitales. El valor del libro y la lectura está construido sobre la base de la utilidad objetiva de esta práctica, pero también sobre creencias subjetivas.

A partir de la información obtenida en campo, se identificaron cinco líneas discursivas que parten del habitus escolar. La primera señala que los libros y la lectura tienen un uso práctico y permiten la educación. La segunda, que estos sirven para el entretenimiento y experimentar emociones. En estos dos casos los libros son 'útiles' en tanto permiten aprender o entretenerse. La tercera línea señala que los libros y la lectura son un camino para la «realización/liberación» personal, la cuarta, que desarrollan habilidades sociales para la participación social, y la quinta, que otorgan legitimidad y permiten diferenciarse de los no lectores. En estas tres últimas líneas discursivas, los libros no solo son «útiles» en tanto permiten «tener algo» sino que aluden a un proceso abstracto de transformación de lo que la persona «es».

En la sociedad escolarizada, los libros y la lectura, incluso aquellos que no tienen un propósito formativo, son "signos» (Baudrillard,1999) de desarrollo cognitivo, sensibilidad, éxito personal, educación y aptitud para el trabajo. Estas apreciaciones no son solo subjetivas, sino que tienen un efecto concreto en las relaciones sociales. Los libros y la lectura invisten a los agentes de legitimidad y no solo les hacen sentirse en una posición de superioridad frente a quienes leen menos o no leen, sino que también les permiten ser reconocidos como mejores.

A manera de conclusión, esta investigación señaló que su principal aporte fue sistematizar y ordenar el sentido común que justifica la importancia del libro y la lectura, incluso en un contexto social donde la desigualdad dificulta el acceso a la «cultura». El mercado de libros Amazonas no es solamente un espacio de intercambios económicos «racionales», sino también de intercambios simbólicos respecto a las ideas, deseos y actitudes hacia el libro, la lectura y la educación.

Este estudio propuso que la distinción social y lo simbólico son dimensiones fundamentales para la construcción de una sociología del libro y la lectura en el Perú, sin embargo, es necesario realizar nuevas investigaciones que presten atención a otros componentes importantes como, por ejemplo, los contenidos de los libros, el efecto de los roles de género en los hábitos de lectura, la lectura de libros en espacios diferentes al mercado Amazonas o incluso las muchas otras formas en que se lee (no necesariamente libros). 
El libro y la lectura han tenido un espacio importante en la identidad e historia peruana; no es gratuito que muchos de los personajes ilustres hayan sido escritores o gente relacionada de una u otra forma a los libros. Esta investigación consideró que una mirada hacia la «Feria de libros del Jirón Amazonas», la cual fue denominada como «El paraíso de los libros» por Mario Vargas Llosa, podría iniciar una reflexión que apunte a entender el lugar que han ocupado los elementos de la «ciudad letrada» (Rama 1998) en lo que consideramos valioso como nación. 


\section{Referencias bibliográfícas}

Baker, D. (2014). The Schooled Society. Stanford: Stanford University Press.

Baudrillard, J. (1999). Crítica de la economía política del signo. Madrid: Siglo Veintiuno.

Berggren, A. (2004). Reading like a Woman. En Reading Sites: Social difference an reader response. New York: Modern Language Association.

Bourdieu, P. (1988a). El interés sociológico. En Cosas dichas (pp. 108-114). Buenos Aires: Gedisa.

Bourdieu, P. (1988b). Espacio social y poder simbólico. En Cosas dichas. Buenos Aires: Gedisa.

Bourdieu, P. (1990). Sociología y cultura. Espacio social y génesis de las clases. México: Grijalbo.

Bourdieu, P. (1991). El sentido práctico. Madrid: Taurus.

Bourdieu, P. (1996a). Consommation culturelle. En Encyclopadia Universalis (pp. 3-44a). París: Encyclopædia Britannica, Inc.

Bourdieu, P. (1996b). Sobre la televisión. Barcelona: Editorial Anagrama.

Bourdieu, P. (1999). La distinción: Criterios y bases sociales del gusto. Madrid: Taurus.

Bourdieu, P. (2000b). Poder, derecho y clases sociales. Bilbao: Desclée de Brouwer.

Bourdieu, P. (2003). Un arte medio: ensayo sobre los usos sociales de la fotografía. Barcelona: Editorial Gustavo Gili, SA.

Bourdieu, P. (2006). Razones prácticas: sobre la teoría de la acción. Barcelona: Editorial Anagrama.

Bourdieu, P., \& Wacquant, L. (1995). Respuestas por una Antropología reflexiva. México: Grijalbo.

Cerlarlc. (2017). Estudio diagnóstico del sector editorial del Perú. Lima: Cámara Peruana del Libro.

Chartier, A.-M., \& Hébrard, J. (1994). Discursos sobre la lectura (1880 - 1980). Barcelona: Editorial Gedisa.

Chartier, R. (1994). The Order of Books: Readers, Authors and Libraries in Europe between the Fourteenth and Eighteenth Centuries. Cambrige: Polity.

García Canclini, N. (1993). El consumo cultural y su estudio en México: una propuesta teórica. En El consumo cultural en México.

García Canclini, N. (1995). Consumidores y Ciudadanos: Conflictos multiculturaes de la globalización. México: Editorial Grijalbo. 
García Canclini, N. (2004). Diferentes, desiguales y desconectados: mapas de la interculturalidad. Barcelona: Gedisa.

García Canclini, N., \& Moneta, C. J. (1999). Las industrias culturales en la integración latinoamericana. México, D.F.: Unesco: Grijalbo: SELA, 1999.

Gutiérrez, A. (2005). LAS PRACTICAS SOCIALES: una introducción a Pierre Bourdieu. Córdoba: Ferreyra Editor.

Huisa, E. (2004). La piratería de libros: problemática para la industria editorial en el Perú. Lima: Tesis digitales UNMSM.

Instituto de Opinión Pública PUCP. (2015). Boletín $N^{\circ}$ 137: LIBROS Y HÁBITOS DE LECTURA. (137), 1-14.

Lahire, B. (2005). Campo, fuera de campo, contracampo. En El trabajo sociológico de Pierre Bourdieu: deudas y críticas (pp. 27-70). Buenos Aires: Siglo XXI Editores Argentina.

Lahire, B., \& Chartier, A.-M. (2004). Sociología de la lectura. Barcelona : Editorial Gedisa, 2004.

Montañez, S., \& Huanca, H. (1999). Mercado de libros en el jirón Amazonas: Sus prioridades. Mercado de libros Amazonas.

Organización de Estados Iberoamericanos. (2014). Encuesta Latinoamericana de hábitos y prácticas culturales. Madrid: Organización de Estados Iberoamericanos para la Educación, la Ciencia y la Cultura (OEI).

Passeron, J.-C. (1991). Le Raisonnement sociologique. En L’space non-poppérien du raisonnement naturel. París: Nathan.

Passeron, J.-C., \& Bourdieu, P. (1970). La Repoduction. París: Ed. de Minuit.

Promolibro. (2006). El libro en el Perú: evolución y diagnóstico, 1995-2005. Lima: Promolibro.

Ragas, J. (2007). Leer, escribir, votar. Literacidad y cultura política en el Perú (18101900). (Spanish). Histórica (02528894), 31(1), 107-134.

Rama, Á. (1998). La ciudad letrada. Montevideo: Arca S.R.L.

Silva-Santisteban, R. (2007). Fotocopias: Por una política cultural latinoamericana. En Industrias culturales: Máquina de deseos en el mundo contemporáneo. Lima: Instituto de Estudios Peruanos.

Vich, V. (2010). El discurso de la calle: los cómicos ambulantes y las tensiones de la modernidad en el Perú. Lima: Red para el Desarrollo de las Ciencias Sociales en el Perú.

Villanueva, C. (1997). El problema del libro en el Perú: El punto de vista de las bibliotecas. Temas de Bibliotecología e Inforación, (1).

Villanueva Montalvo, A. (2004). El circuito informal de libros en el Perú: El caso de la Cámara Popular de Libreros del Jirón Amazonas. Biblios, 5(17), 3-15. 